\title{
Differences in Symptoms among Adults with Canal versus Otolith Vestibular Dysfunction: A Preliminary Report
}

\author{
Lisa Farrell ${ }^{1,2}$ and Rose Marie Rine ${ }^{3}$ \\ ${ }^{1}$ Adjunct Clinical Faculty, Nova Southeastern University, 3200 South University Drive, Fort Lauderdale, FL 33328, USA \\ ${ }^{2}$ OrthoSport, Inc., 5200 South University Drive, Suite 105, Fort Lauderdale, FL 33328, USA \\ ${ }^{3}$ Specialty Therapy Source LLC, 12948 Palmetto Glade Drive, Jacksonville, FL 32246, USA \\ Correspondence should be addressed to Lisa Farrell; adventurefour@aol.com
}

Received 28 October 2013; Accepted 26 November 2013; Published 9 January 2014

Academic Editors: S. Eyigor and C.-L. Kao

Copyright $\odot 2014$ L. Farrell and R. M. Rine. This is an open access article distributed under the Creative Commons Attribution License, which permits unrestricted use, distribution, and reproduction in any medium, provided the original work is properly cited.

\begin{abstract}
Despite the importance of symptomatology in the diagnosis of vestibular dysfunction, the qualitative nature of the symptoms related to semicircular canal (canal) versus otolith dysfunction is not fully understood. The purpose of this study was to compare symptoms, and their severity, in individuals with canal versus otolith peripheral vestibular dysfunction. A subjective tool, the Descriptive Symptom Index (DSI), was developed to enable categorization of symptoms as rotary, linear, imbalance or falls, and nondistinct. Fourteen adults were recruited and grouped based on vestibular function testing: canal only dysfunction, otolith only dysfunction, or canal and otolith dysfunction. Also, the Dizziness Handicap Inventory (DHI) was used to grade the severity of perceived limitations due to symptoms. The DSI was reliable and differentiated those with canal (rotary symptoms) versus otolith (linear symptoms) dysfunction. Most individuals with otolith only dysfunction did not report rotary symptoms. DHI scores were significantly higher in those with otolith dysfunction, regardless of canal functional status. All who experienced falls had otolith dysfunction and none had canal only dysfunction. Results support the importance of using linear and rotary descriptors of perceived disorientation as part of diagnosing vestibular dysfunction.
\end{abstract}

\section{Introduction}

A comprehensive patient history, which includes the qualitative nature of symptoms, is paramount when making the diagnosis of peripheral vestibulopathy $(\mathrm{P}-\mathrm{VeD})$ in adults who report dizziness and/or imbalance [1-3]. Rotary vertigo has traditionally been accepted as the primary descriptor related to $\mathrm{P}-\mathrm{VeD}[2,4-6]$. The diagnosis is often confirmed with objective measurement of the functional integrity of the semicircular canals (canals) using calorics, rotational chair, head impulse, and/or Dix-Hallpike testing [4]. However, the clinical diagnostic process has been limited because measurement of otolith function has not been readily available. Also, the qualitative nature of the symptoms of otolith dysfunction has not been formally investigated and determined $[7,8]$. Tomanovic and Bergenius [3], who studied the prevalence of different types of dizziness symptoms in subjects with $\mathrm{P}-\mathrm{VeD}$, expanded the understanding of subjective descriptors by concluding that in addition to the classic symptoms of vertigo, the presence of "nonclassical" symptoms, such as drop attacks, unsteadiness like walking on a boat, walking on pillows, stepping into a hole, and feeling like being pulled to one side, occur in these individuals. In Tomanovic's study, only tests of canal function, and not otolith function, determined the diagnosis of P-VeD. It is not known whether the otolith organs (e.g., utricle and saccule) were also damaged and may have contributed to the subjects' symptoms, especially the "nonclassical" symptoms.

Advances in vestibular function testing procedures has identified otolith dysfunction [6,9-11] and several investigations have reported that subjects with otolith dysfunction described their symptoms as feeling like sensations of rocking, tilting, walking on pillows, being pushed or pulled, feeling drunk, and falling $[7,12]$. Even though these descriptions were only anecdotally associated with $\mathrm{P}-\mathrm{VeD}$, it questions whether the limited focus on symptoms of rotary vertigo 
impedes the diagnostic process. Consequently, by focusing on rotary vertigo as the symptom associated with P-VeD, successful management, including selection of appropriate vestibular rehabilitation (VR) strategies, may be hindered. The advancement of clinical practice for individuals with complaints of dizziness and/or imbalance requires valid and reliable testing of canal and the otolith end-organ function, confirmation of the selective effect of pathology on canal versus otolith mechanisms, improved understanding of how the unique functional contributions of canal and otolith mechanisms affects perceived orientation and balance ability after substrate damage, and expansion of the descriptors used by individuals with vestibular dysfunction to help with the diagnostic process and with establishing the need for VR.

Recently, there have been improvements in clinical practice with the expansion from testing only canal function (specifically of the horizontal canal) to the emergence of testing of otolith function $[5,6,13-16]$. Tests of utricular function are the subjective visual vertical test (SVV; both static and dynamic) [13] and the ocular vestibular evoked myogenic potential (o-VEMP) $[13,15]$. The integrity of the saccule and the inferior branch of the vestibular nerve function is measured by using the cervical vestibular evoked myogenic potential (c-VEMP) $[5,13]$. As a consequence of the expansion of vestibular function testing, the understanding of otolith dysfunction is emerging. Traditionally, it has been assumed that canal and otolith mechanisms would not be selectively affected by disease or injury, which would argue against the need for testing all mechanisms of the vestibular system [4]. By utilizing tests of canal and otolith function, results of investigations have shown that involvement of these mechanisms can be discriminative and supports the importance of comprehensive testing. Karlberg et al. [14] presented the cases of two patients diagnosed with superior vestibular neuritis based on abnormal caloric, abnormal SVV, and normal c-VEMP tests, thus indicating both canal and otolith involvement. Halmagyi et al. [17] and Monstad et al. [10] reported that their patients with inferior vestibular neuritis presented with symmetrical calorics, normal head impulse testing of the anterior and horizontal canals, abnormal head impulse tests for the posterior canal, and abnormal c-VEMP results. Similarly, Iwasaki et al. [9] found that patients with Meniere's disease, acoustic neuroma (AN), cerebellopontine angle tumor other than AN, sudden deafness with vertigo, and multiple sclerosis can present with involvement of only the inferior vestibular nerve as measured by normal caloric testing and abnormal c-VEMP testing. According to Kingma and Wit [18], for patients with Meniere's disease, saccular dysfunction is an established pathophysiological feature and explains why abnormal c-VEMP test results are reported. However, other authors report that the results are dependent upon the phase and the stage of the disease [19-21]. Manzari et al. [20] demonstrated that utricular and saccular function is affected differently in patients with Meniere's disease of less than two-year duration. There was a significant increase in o-VEMP response during an active phase as compared to the quiescent phase, but a significant decrease in cVEMP response during the active phase as compared to quiescence. Young et al. [21] reported that c-VEMP amplitude responses varied depending on the stage of the disease with a significant increase in the asymmetry ratio as the disease progressed. Additionally, de Waele et al. [19] reported that c-VEMP responses were absent in 54\% of patients tested in the quiescent period of Meniere's disease (stage not reported) and that c-VEMP results did not correlate with canal paresis. Similar to these reports of saccular pathology, utricular dysfunction has also been reported in patient cases with or without concurrent canal and saccule involvement. Tabak et al. [22] found that a group of patients who had vestibular loss after surgery for acoustic neuroma were deficient in canal and otolith function as measured by calorics and SVV tests. Results of the investigation by Schönfeld et al. [6] also provide evidence of isolated unilateral utricular dysfunction, as demonstrated by asymmetric dynamic SVV results, but symmetric caloric and c-VEMP responses in patients with chronic disease. Despite advances in the development of clinical vestibular function testing and the identification of isolated otolith dysfunction, a comprehensive comparative investigation of the qualitative nature of the symptoms has not been done.

The neurophysiology of the canals and otolith organs is distinct with unique contributions of each to perceived head position and balance abilities. Therefore, adults with dizziness and imbalance due to otolith organ involvement may present with different symptoms as compared to those with canal dysfunction. Both canals and otolith organs contribute to the perception of head orientation, but each responds to unique stimuli [23-25]. The canals are optimally sensitive to angular acceleration, which explains why patients with pathology affecting the canals report symptoms of spinning (rotary vertigo) [7]. The otolith organs, although less thoroughly studied and understood $[8,12]$, optimally respond to linear and gravitational acceleration due to translational head movements and head tilts $[2,24]$. This could explain why anecdotal reports of feeling like rocking, tilting, walking on pillows, being pushed, feeling drunk, and falling have been used by patients with otolith dysfunction $[7,12]$. In addition to perceived orientation, canals and otolith organs both contribute to postural control via vestibulospinal pathways. However, it is thought that the contribution of the otoliths is the main vestibular source for postural control $[23,25]$. The vestibulospinal pathways that receive canal input terminate on motor neurons of the cervical cord, whereas those of the otolith organs innervate motor neurons from the cervical through the sacral cord [26]. The otolith organ input facilitates activation of antigravity muscles and modulates the tone of neck, trunk, and limb extensor muscles enabling the maintenance of posture [25]. Consequently, it seems logical to conclude that dysfunction of canals versus that of otolith organs would effect perceived orientation and balance differently. In turn, it would seem reasonable that exercise strategies used in rehabilitation would need to be designed specifically to promote stimulation of residual function of these structures.

Vestibular rehabilitation (VR) exercises, which focus on the reduction of complaints of dizziness and imbalance, have been based on diagnoses involving only the canals. VR has emphasized the use of rotational head movement 
exercises, which optimally stimulates canal function [27]. This may affect the success of intervention because these exercises do not optimally stimulate the otolith organs, and thus, the exercises used may be incomplete or incorrect for individuals with otolith dysfunction. This could explain, at least in part, the variable outcomes of VR that are reported $[28,29]$. A prospective, controlled study by Krebs et al. [29] demonstrated that only $60 \%$ of patients with unilateral and bilateral P-VeD who performed gaze stability and balance retraining exercises benefited from the VR as indicated by improved gait parameters. Ernst et al. [28] reported that although there was improvement of perceived orientation, gaze stability, and/or postural control following VR in most patients with vestibulopathy, some patients did not have resolution of their symptoms. A prospective study by Enticott et al. [30] found that patients with a mixture of vestibular dysfunctions (canal only, canal and otolith, and otolith only) who performed a variety of head turning and walking tasks over 10 weeks had significant improvement of impairments compared to patients who only performed lower extremity strengthening exercises. Even though perceived disability, balance confidence, and severity of symptoms improved in patients studied by Enticott, patients' perception of the therapy program's impact on their symptoms did not improve significantly in the treatment group compared to the control group. By having a more thorough understanding of the qualitative nature of the symptoms of canal versus otolith dysfunction, the diagnosis could be more specific, which could improve the development of optimal intervention for individuals with vestibulopathy. However, to date, this has not been done.

The primary objective of this investigation was to identify the qualitative nature of dizziness and imbalance and determine the impact of these symptoms on daily activities in adults with canal versus otolith $\mathrm{P}-\mathrm{VeD}$. This information can positively impact the selection of diagnostic tests, improve diagnosis, and lead to the development of improved rehabilitation strategies.

\section{Methods}

Fourteen subjects, aged 36-59 years (mean $=46$; $s d=$ 5.8) with complaints of dizziness and/or imbalance due to a diagnosis of $\mathrm{P}-\mathrm{VeD}$, were recruited from the patient population seen at the University of Miami Miller School of Medicine's Department of Otolaryngology. Individuals were excluded from the study if they had a cognitive deficit or a confounding diagnosis, such as orthopedic injury or condition involving the spine or lower extremities; neurological, somatosensory, or visual dysfunction due to a CNS lesion; peripheral neuropathy of the legs; or spinal cord injury. Diagnosis was confirmed by history, neurootologic examination, and vestibular function testing (e.g., horizontal head impulse/thrust, rotational chair, calorics, DixHallpike, SVV, and c-VEMP). Diagnoses included acoustic neuroma resection, benign paroxysmal positional vertigo (BPPV), Meniere's disease, superior canal dehiscence, unilateral labyrinthitis, and neuritis. The range of time from symptom onset was 3 weeks to 39 months (mean $=16$; $\mathrm{sd}=$ 13.5).

Subjects were grouped based on test results: Group 1 had abnormal canal testing and normal otolith testing $(n=3$; $21 \%)$, Group 2 had abnormal otolith testing and normal canal testing ( $n=4 ; 29 \%)$, and Group 3 had abnormal canal and abnormal otolith testing $(n=7 ; 50 \%)$. See Table 1 for a summary of subject demographics and group assignment.

\section{Instrumentation}

A tool, the Descriptive Symptom Index (DSI), was developed to enable examination of symptoms reported. The DSI enabled categorization of subjects' perception of dizziness and imbalance symptoms. To minimize bias, subjects were first asked by an investigator (R.M.R.), who was blinded to vestibular function test results, to describe their symptoms. They were then provided a list of potential symptoms and asked to indicate whether the word did or did not further clarify their symptoms (Table 2). The descriptors provided or selected by subjects were recorded and categorized (rotary sensations for category 1, linear sensations for category 2, symptoms that portray imbalance and falls for category 3 , or nondistinct symptoms for category 4). Categorization was used to examine the relationship between the qualitative nature of symptoms and vestibular mechanism involved and to compare symptoms between groups. Retest was completed at the end of initial visit to enable examination of test-retest reliability of the DSI.

To quantify the severity of perceived limitations with daily activities, the Dizziness Handicap Inventory (DHI) was completed. This test is made up of 25 questions that address the emotional, physical, and functional aspects regarding how symptoms of dizziness and/or imbalance impact daily activities. The DHI has been shown to provide high testretest reliability $(r=0.97)$ [31], high internal consistency (Cronbach alpha $=0.89$ ) [19], and there is evidence for construct validity with significant correlation between balance confidence and specific measures of balance [32]. Subjects were asked to indicate "yes," "sometimes," or "no" as it pertained to how their dizziness and/or imbalance limited their function. Answers were assigned 4 points for "yes," 2 points for "sometimes," or 0 points for "no." The composite score ranged from 0 (no perceived handicap) to 100 (maximum perceived handicap). Based on the report by Whitney et al. [32], a severity score was assigned based on the composite score: (1) mild handicap ranged from 0 to 30 , (2) moderate handicap ranged from 31 to 60 , and (3) severe handicap ranged from 61 to 100 . The DHI composite and severity scores were used to compare groups' perceived handicap due to dizziness and/or imbalance.

\section{Analysis}

Statistical analyses were performed using the SPSS software package (SPSS Inc., 233 S. Wacker Drive, Chicago, IL). The reliability of the DSI was calculated using a kappa statistic. Sensitivity, specificity, positive predictive value (PPV), and 
TABLE 1: Subject demographics and group assignment.

\begin{tabular}{|c|c|c|c|c|c|c|c|c|c|c|}
\hline \multirow[t]{2}{*}{$\begin{array}{l}\begin{array}{l}\text { Age } \\
\text { (yrs) }\end{array} \\
\end{array}$} & \multirow[t]{2}{*}{ Gender } & \multirow[t]{2}{*}{ Group } & \multicolumn{4}{|c|}{ Canal function tests } & \multicolumn{2}{|c|}{ Otolith function tests } & \multirow[t]{2}{*}{$\begin{array}{c}\text { Diagnosis(es) } \\
\text { established by physician }\end{array}$} & \multirow[t]{2}{*}{$\begin{array}{c}\text { Onset } \\
\text { (months) }\end{array}$} \\
\hline & & & $\mathrm{DH}$ & $\mathrm{R}$ & $\mathrm{C}$ & HT & SVV & VEMP & & \\
\hline 36 & Female & 1 & - & - & + & + & - & - & Vestibular labyrinthitis & 2 \\
\hline 51 & Female & 1 & + & - & - & - & - & - & BPPV & $3 / 4$ \\
\hline 53 & Female & 1 & - & + & - & + & - & - & Vestibular neuritis & 15 \\
\hline 44 & Male & 2 & NT & - & NT & - & - & + & Vestibular neuritis & 12 \\
\hline 44 & Female & 2 & - & - & - & - & + & + & Vestibular neuritis & 12 \\
\hline 48 & Female & 2 & - & - & - & - & + & - & Vestibular neuritis & 36 \\
\hline 50 & Female & 2 & - & - & - & - & + & + & Meniere's disease & 6 \\
\hline 46 & Male & 3 & - & NT & + & + & + & - & Meniere's disease & 36 \\
\hline 40 & Male & 3 & + & - & + & + & + & + & BPPV and labyrinthitis & 18 \\
\hline 43 & Female & 3 & + & NT & + & + & + & + & $\begin{array}{l}\text { BPPV and acoustic } \\
\text { Neuroma resection }\end{array}$ & 1 \\
\hline 43 & Female & 3 & + & NT & + & - & - & + & $\begin{array}{l}\text { BPPV and vestibular } \\
\text { neuritis }\end{array}$ & 39 \\
\hline 40 & Female & 3 & NT & + & + & + & + & + & $\begin{array}{c}\text { Endolymphatic sac decomp } \\
2^{\circ} \text { Meniere's }\end{array}$ & 5 \\
\hline 59 & Female & 3 & - & + & - & + & + & + & Superior canal dehiscence & 24 \\
\hline 47 & Female & 3 & + & - & - & - & + & + & $\begin{array}{l}\text { BPPV and vestibular } \\
\text { neuritis }\end{array}$ & 24 \\
\hline
\end{tabular}

DH: Dix-Hallpike; R: rotary chair; C: calorics; HT: head thrust; SVV: subjective visual vertical; VEMP: vestibular evoked myogenic potential; NT: not tested; BPPV: benign paroxysmal positional vertigo.

TABLE 2: Descriptive symptom index.

\begin{tabular}{llll}
\hline Category 1 & Category 2 & Category 3 & Category 4 \\
\hline Tumbling (self) & Rocking (self) & Off balance & Lightheadedness \\
Tumbling (world) & Rocking (world) & Unsteady & Floating \\
Spinning (self) & Tilting (self) & Staggers & Disoriented \\
Spinning (world) & Tilting (world) & Falls & Swimming \\
Other & Pulling & Other & Wooziness \\
& Pushing & & Other \\
& Linear motion (self) & & \\
& Linear motion (world) & & \\
& Other & & \\
\hline
\end{tabular}

negative predictive value (NPV) for the DSI categories 1 (rotary symptoms) and 2 (linear symptoms) were calculated to determine whether these categories were able to discriminate between subjects with canal versus otolith dysfunction. Chi-square statistics were used to compare DSI categories between groups. An ANOVA statistic with Tukey HSD post hoc tests was utilized to compare DHI scores between groups and Kruskal-Wallis statistic was calculated to compare severity rank (mild, moderate, and severe) of DHI scores between groups.

\section{Results}

An a priori power analysis revealed that, to achieve a power level of $60 \%$ with a medium effect size and a significance level of 0.05 , a sample size of 23 subjects per group was required. Since this sample size was not achieved, an alpha level of 0.10 was used to determine trends in the data. The fair to excellent sensitivity of DSI categorization supports this approach.

Time from symptom onset did not correlate with severity of symptoms $(P=0.359, r=0.27)$. Although there was no difference in time from symptom onset between groups $(P=0.29)$, there was a trend evident in that Group 3 had the greatest time from symptom onset (Figure 1).

DSI scores had good to excellent test-retest reliability for each symptom category: rotary symptoms $\kappa=1.0$, linear symptoms $\kappa=0.63$, imbalance and falls $\kappa=1.0$, and nondistinct symptoms $\kappa=1.0$. The good to excellent sensitivity and positive predictive value suggested that the DSI's rotary and linear categorizations (Categories 1 and 2, resp.) were able to 


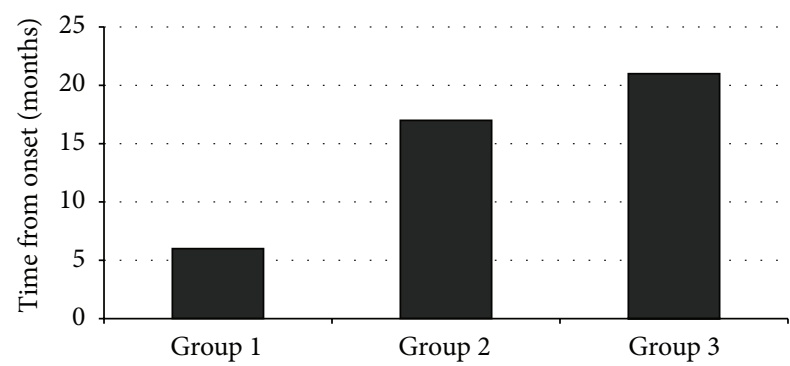

FIGURE 1: Comparison of symptom onset time between groups. Although the difference was not significant, time from symptom onset was greatest in Group 3 (otolith and canal dysfunction), as compared to those in Group 1 (canal only dysfunction) or Group 2 (otolith only dysfunction).

TABLE 3: Sensitivity, specificity, and predictive values for rotary and linear symptoms for subjects with P-VeD.

\begin{tabular}{lcc}
\hline & $\begin{array}{c}\text { Rotary symptoms and } \\
\text { canal dysfunction } \\
\text { (DSI category 1) }\end{array}$ & $\begin{array}{c}\text { Linear symptoms and } \\
\text { otolith dysfunction } \\
\text { (DSI category 2) }\end{array}$ \\
\hline Sensitivity & $80 \%$ & $100 \%$ \\
Specificity & $75 \%$ & $33 \%$ \\
PPV & $89 \%$ & $85 \%$ \\
NPV & $60 \%$ & $100 \%$ \\
\hline
\end{tabular}

P-VeD: peripheral vestibulopathy; DSI: Descriptive Symptom Index; PPV: positive predictive value; NPV: negative predictive value.

correctly classify individuals' involved vestibular mechanism (Table 3).

It should be noted that one of the four individuals with otolith only dysfunction (Group 2) reported rotary symptoms and one of the three individuals with canal only dysfunction (Group 1) reported linear symptoms. There were group differences in frequency of reported rotary symptoms $(P=$ 0.01 ) with Group 3 reporting rotary symptoms significantly more often than Group $1(P=0.008)$ and Group $2(P=0.004)$ (Table 4). It was also evident that there was a trend in the difference in frequency of reported linear symptoms between groups $(P=0.07)$ with Group 3 reporting more linear symptoms than Group $1(P=0.05)$. Consistent with these findings, when individuals were grouped by the presence or absence of vestibular substrate pathology, those with canal dysfunction (Groups 1 and 3) reported rotary symptoms more often than those without canal dysfunction (Group 2; $P=$ 0.03 ) and those with otolith dysfunction (Groups 2 and 3) reported linear symptoms more often than those without otolith dysfunction (Group 1; $P=0.02$ ) (Tables 5 and 6).

Chi-square statistic was not calculated for the imbalance and falls category (category 3 ) nor for the indistinct category (category 4) of the DSI since all subjects within each group reported these symptoms. The majority of subjects described their balance difficulties as being off balance $(100 \%)$ and unsteady (100\%). Forty-three percent of subjects had a history of falls, and all of the fallers were individuals with otolith dysfunction (Groups 2 and 3) (Figure 2).
TABLE 4: Frequency of reported rotary and linear symptoms for each $\mathrm{P}-\mathrm{VeD}$ group.

\begin{tabular}{lcccc}
\hline & $\begin{array}{c}\text { Group 1 } \\
(n=3)\end{array}$ & $\begin{array}{c}\text { Group 2 } \\
(n=4)\end{array}$ & $\begin{array}{c}\text { Group 3 } \\
(n=7)\end{array}$ & $P$ value \\
\hline $\begin{array}{l}\text { Rotary } \\
\text { (DSI cat. 1) }\end{array}$ & $1(33.3 \%)$ & $1(25 \%)$ & $7(100 \%)$ & $0.01^{*}$ \\
$\begin{array}{l}\text { Linear } \\
\text { (DSI cat. 2) }\end{array}$ & $2(66.7 \%)$ & $4(100 \%)$ & $7(100 \%)$ & $0.07^{*}$ \\
\hline
\end{tabular}

P-VeD: peripheral vestibulopathy; Group 1: canal only dysfunction; Group 2: otolith only dysfunction; Group 3: both.

${ }^{\S}$ Chi-square statistic.

${ }^{*} \alpha=0.10$.

TABLE 5: Frequency of reported rotary and linear symptoms for those with the presence or absence of canal dysfunction.

\begin{tabular}{lccc}
\hline & $\begin{array}{c}\text { Canal } \\
\text { dysfunction } \\
(n=10)\end{array}$ & $\begin{array}{c}\text { No canal } \\
\text { dysfunction } \\
(n=7)\end{array}$ & $P$ value \\
\hline $\begin{array}{l}\text { Rotary } \\
(\text { DSI cat. 1) }\end{array}$ & $8(80 \%)$ & $1(25 \%)$ & $0.03^{*}$ \\
$\begin{array}{l}\text { Linear } \\
\text { (DSI cat. 2) }\end{array}$ & $9(90 \%)$ & $4(100 \%)$ & 0.26 \\
\hline $\begin{array}{l}\text { DSI: Descriptive Symptom Index. } \\
{ }^{\S} \text { Chi-square statistic. } \\
{ }^{*} \alpha=0.10 .\end{array}$
\end{tabular}

TABLE 6: Frequency of reported rotary and linear symptoms for those with the presence or absence of otolith dysfunction.

\begin{tabular}{lccc}
\hline & $\begin{array}{c}\text { Otolith } \\
\text { dysfunction } \\
(n=11)\end{array}$ & $\begin{array}{c}\text { No otolith } \\
\text { dysfunction } \\
(n=3)\end{array}$ & $P$ value \\
\hline $\begin{array}{l}\text { Rotary } \\
\text { (DSI cat. 1) }\end{array}$ & $8(73 \%)$ & $1(33.3 \%)$ & 0.10 \\
$\begin{array}{l}\text { Linear } \\
(\text { DSI cat. 2) }\end{array}$ & $11(100 \%)$ & $2(66.7 \%)$ & $0.02^{*}$ \\
\hline
\end{tabular}

DSI: Descriptive Symptom Index.

${ }^{\S}$ Chi-square statistic.

${ }^{*} \alpha=0.10$.

Interestingly, the majority of subjects from each of the groups reported being disoriented, woozy, nauseated, and feeling like they were floating, and all, except for one subject with canal dysfunction, had feelings of lightheadedness. Although sensations of feeling fatigued (29\%), full headed (14\%-25\%), floor moving (14\%-25\%), drunkenness (25\%), and uncoordinated $(25 \%)$ were used by those with otolith dysfunction (Groups 2 and 3), those with canal only dysfunction (Group 1) never used these words to describe their symptoms (Figure 3 ).

With regards to severity of perceived limitations with daily activities due to symptoms, results of parametric testing revealed that a trend was evident between groups' DHI composite scores $(P=0.09)$ and there were significant differences between groups' physical domain scores $(P=$ 0.01; Table 7). Post hoc testing revealed that Group 2 trended toward higher (worse) composite scores compared to Group $1(P=0.10)$, and Groups 2 and 3 had significantly higher 

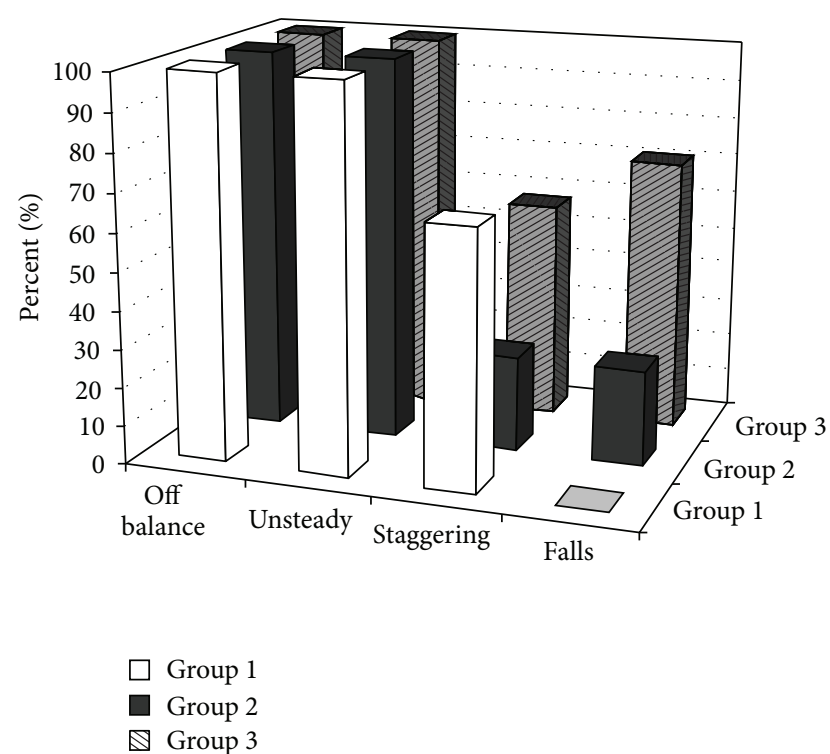

FIGURE 2: Frequency of imbalance and falls symptoms (DSI category 3) between Groups. The percentage of individuals within each Group that reported imbalance symptoms is depicted. All who reported falls had otolith dysfunction (Groups 2 and 3).

TABLE 7: Comparison of groups' DHI scores.

\begin{tabular}{|c|c|c|c|c|}
\hline & $\begin{array}{c}\text { Group 1 } \\
(n=3)\end{array}$ & $\begin{array}{c}\text { Group } 2 \\
(n=4)\end{array}$ & $\begin{array}{c}\text { Group } 3 \\
(n=7)\end{array}$ & $P$ value \\
\hline Composite $^{\dagger}$ & $43(14.5)$ & $64(6.0)$ & $60(17.6)$ & $0.09^{*}$ \\
\hline P-DHI ${ }^{\dagger}$ & $10(6.0)$ & $19(3.0)$ & $20(4.8)$ & $0.01^{*}$ \\
\hline F-DHI ${ }^{\dagger}$ & $17(8.3)$ & $24(4.1)$ & $21(8.0)$ & 0.28 \\
\hline E-DHI ${ }^{\dagger}$ & $15(6.1)$ & $22(5.3)$ & $19(8.4)$ & 0.28 \\
\hline $\operatorname{Mild}^{\dagger \dagger}$ & 1 & 0 & 1 & \\
\hline Moderate $^{\dagger \dagger}$ & 2 & 1 & 2 & 0.18 \\
\hline Severe $^{\dagger \dagger}$ & 0 & 3 & 4 & \\
\hline
\end{tabular}

DHI: Dizziness Handicap Inventory; Group 1: canal only dysfunction; Group 2: otolith only dysfunction; Group 3: both.

${ }^{\dagger}$ Mean DHI scores (standard deviation in parentheses).

P-DHI: physical domain; F-DHI: functional domain; E-DHI: emotional domain.

${ }^{\dagger \dagger}$ Composite score severity rank frequencies.

${ }^{\S}$ ANOVA.

${ }^{*} \alpha=0.10$.

(worse) physical domain scores than those in Group $1(P=$ 0.04 and $P=0.01$, resp.; Figure 4(a)).

If subjects were grouped by the presence or absence of otolith dysfunction, individuals with otolith dysfunction (Groups 2 and 3) did have higher (worse) composite and physical domain DHI scores as compared to those without otolith dysfunction (Group 1; $P=0.03, P=0.004$, resp.; Table 8; Figure 4(b)).

Examination of the frequency of mild, moderate, or severe ranking of DHI scores did not differ between the three groups (Table 7). However, when grouped by those with and without otolith dysfunction, there was a trend for individuals with otolith dysfunction (Groups 2 and 3) to have
TABLE 8: Comparison of those with and without otolith dysfunctionDHI scores.

\begin{tabular}{cccc}
\hline & $\begin{array}{c}\text { Otolith } \\
\text { dysfunction } \\
(n=11)\end{array}$ & $\begin{array}{c}\text { Normal otolith } \\
\text { function } \\
(n=3)\end{array}$ & $P$ value \\
\hline Composite $^{\dagger}$ & $61(14.1)$ & $43(14.5)$ & $0.03^{*}$ \\
P-DHI $^{\dagger}$ & $20(4.2)$ & $10(6.0)$ & $0.004^{*}$ \\
F-DHI $^{\dagger}$ & $22(6.7)$ & $17(8.3)$ & 0.17 \\
E-DHI $^{\dagger}$ & $19(7.2)$ & $17(6.1)$ & 0.17 \\
Mild $^{\dagger \dagger}$ & 1 & 1 & \\
Moderate $^{\dagger \dagger}$ & 3 & 2 & $0.07^{*}$ \\
Severe $^{\dagger \dagger}$ & 7 & 0 & \\
\hline
\end{tabular}

DHI: Dizziness Handicap Inventory.

${ }^{\dagger}$ Mean DHI scores (standard deviation in parentheses).

P-DHI: physical domain; F-DHI: functional domain; E-DHI: emotional domain.

${ }^{\dagger \dagger}$ Composite score severity rank frequencies.

${ }^{\S}$ ANOVA.

${ }^{*} \alpha=0.10$.

DHI rankings that were more severe as compared to those without otolith dysfunction (Group 1; $P=0.07$; Table 8). All individuals with otolith dysfunction (Groups 2 and 3), except one, had DHI rankings that were categorized as either moderate or severe, whereas those without otolith dysfunction (Group 1) had DHI rankings that were categorized as either mild or moderate.

\section{Discussion}

Evidence from this preliminary investigation confirmed that the qualitative nature of dizziness and the severity of perceived limitations with daily activities due to these symptoms vary in adults with report of dizziness and/or imbalance depending upon the involved pathological vestibular mechanism. Symptoms reported by those with canal dysfunction (Groups 1 and 3) were predominantly rotary in nature. These individuals used words like "spinning" and "tumbling" to describe their symptoms, whereas those with otolith dysfunction (Groups 2 and 3) reported symptoms that were predominantly linear in nature by using words like "tilting," "pulling," "pushing," "floor falling away," and/or "rocking to and fro" or "rocking back and forth" to describe their symptoms. These types of symptoms were also reported anecdotally in studies by Brandt [7] and Basta et al. [12]. The results reported here, that most individuals with only otolith dysfunction did not report rotary-type symptoms, argue for the inclusion of linear descriptors of symptoms in the diagnostic process. Furthermore, not only did most individuals with isolated pathology (Group 1 and Group 2) report distinct symptomology that was expected for their pathology, all individuals with both canal and otolith dysfunction (Group 3) reported both rotary-type and lineartype symptoms. This information is invaluable since patient history remains of the utmost importance when determining whether symptoms of dizziness and/or imbalance are due to $\mathrm{P}-\mathrm{VeD}$ [2]. It can also improve the diagnostic process 


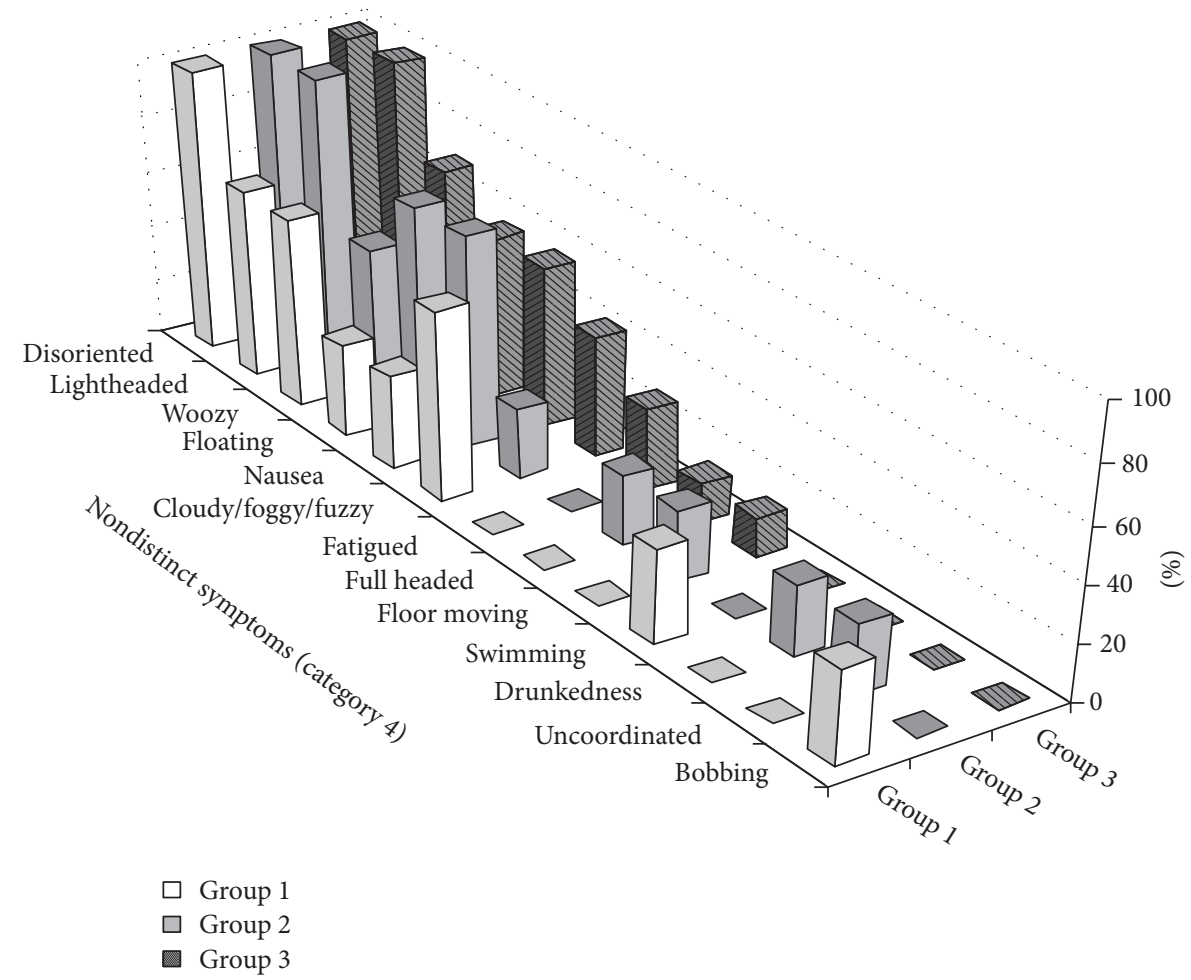

FIGURE 3: Frequency of nondistinct symptoms (DSI category 4) between Groups. Interestingly, most subjects in each of the groups (Group1 = canal only dysfunction; Group 2 = otolith only dysfunction; Group 3 = otolith and canal dysfunction) reported nondistinct symptoms, such as lightheaded, disorientation, and feeling woozy, but several were unique to those with otolith only dysfunction (e.g., full headed, floor moving, feeling drunk, and uncoordinated) and a couple were unique to those with canal only dysfunction (e.g., swimming, bobbing).

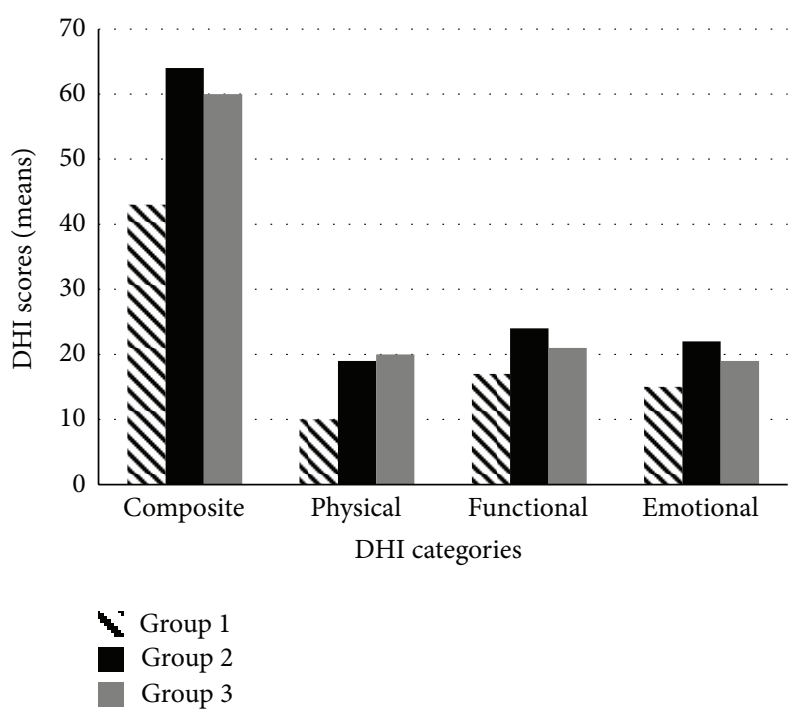

(a)

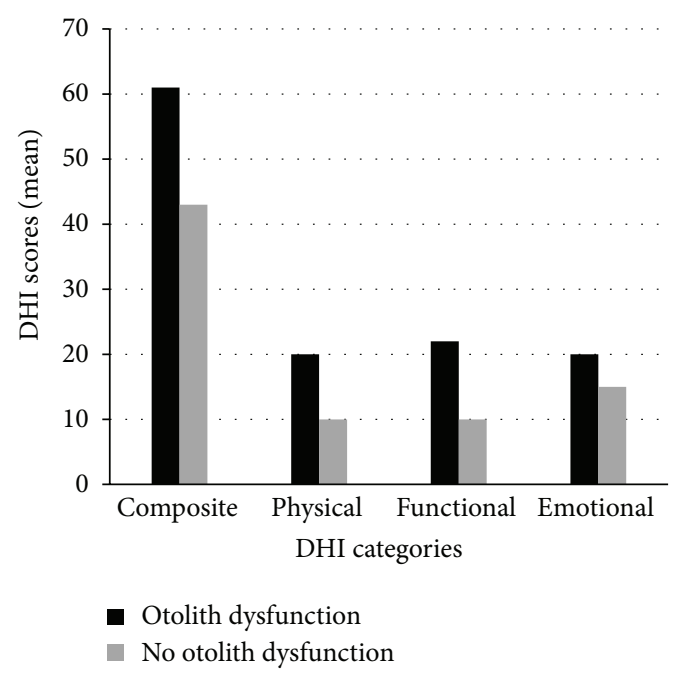

(b)

FIGURE 4: (a) Comparison of Dizziness Handicap Inventory (DHI) scores between groups. All DHI scores were higher (worse) for Groups 2 (otolith only dysfunction) and 3 (otolith and canal dysfunction) as compared to Group 1 (canal only dysfunction), most notably on the composite scores $(P=0.09)$ and physical domain scores $(P=0.01)$. (b) Comparison of DHI scores between those with and without otolith dysfunction. Comparison of composite and physical scores revealed that those with otolith dysfunction (Groups 2 and 3 ) had significantly worse scores as compared to those with canal only dysfunction (Group 1) $(P=0.03$ and $P=0.004$, resp.). This suggests that those with otolith dysfunction perceive their symptoms to be more disruptive of their life style as compared to those with canal only dysfunction. 
by guiding the physical examination and aid in deciding which vestibular function tests are needed to confirm the diagnosis [1]. Improvement of the diagnostic process can lead to better management of adults with complaints of dizziness and imbalance by referring them to VR.

Whereas specific symptoms related to rotary- and lineartype symptoms (DSI categories 1 and 2) were associated with the involved vestibular mechanism, symptoms related to those of imbalance (DSI category 3 ) and those that were nondistinct (category 4) did not help distinguish the type of involved vestibular mechanism. All individuals reported that they felt "off balance," "unsteady," and "disoriented." Although imbalance and nondistinct descriptors were not helpful in identifying involved P-VeD mechanism, the information should not be disregarded. This information identifies the need for further assessment, particularly evaluation of possible postural control impairments. Although ambiguous reports of imbalance did not identify the involved vestibular mechanisms, reports of falls in DSI category $3 \mathrm{did}$ demonstrate apparent differences between $\mathrm{P}-\mathrm{VeD}$ groups. No individuals with canal only dysfunction reported falls. This may be attributed to the neurophysiological role that the otolith organs play in modifying tone in antigravity muscles for postural control. Additional support for this theory was demonstrated with the greater severity of perceived limitations with daily activities, as measured by DHI, in individuals who had otolith dysfunction, regardless of the presence of canal dysfunction (Groups 2 and 3). In particular, the physical domain scores were significantly higher (worse) scores when all individuals were grouped based on presence of otolith dysfunction, regardless of whether or not the individuals had canal dysfunction (Groups 2 and 3), as compared to those with canal only dysfunction (Group 1). Walking in the market, walking down a sidewalk, and bending over were the items within the physical domain of the DHI that demonstrated the most differences. The findings of greater subjective reports of falls and the limitations with daily activities in this study are supported by evidence of objective impairments of postural control in those with reduced otolith function. Basta et al. [12] demonstrated that sensory organization testing and trunk sway measures during stance and gait activities were impaired in those with otolith only dysfunction as compared to healthy controls. Serrador et al. [33] demonstrated that with age there is a reduction of utricular function as measured by ocular counter rolling, and as a result, individuals can have increased postural sway and be at increased risk of falling. Interestingly, the difference in the severity of perceived limitations with daily activities reported in this study is in contrast to that reported by Murray et al. [34]. These investigators found no difference in DHI scores between subjects who had canal only dysfunction (positive calorics) as compared to those who had canal and otolith dysfunction (positive calorics, positive VEMP, and/or SVV). Although this study and the Murray et al. [34] study were similar by using the same vestibular function tests to confirm the diagnosis of subjects with chronic complaints of dizziness and/or imbalance, differences in the subject sampling may explain the differences in DHI findings. In the current study, several subjects with otolith only dysfunctions were included and they were not in the Murray et al. [34] article. This study provides support for the predominant functional contribution of the otolith organs to postural control, as was reported by Markham [25]. However, further objective comparison of postural control and balance impairments between those with canal versus otolith dysfunction needs to confirm these findings.

Although it was not the primary objective of this investigation, comprehensive vestibular function testing confirmed the selective effect of pathology on vestibular mechanisms, which resulted in distinct canal and otolith pathology or a combination of the two $[4,16]$. In this investigation, seven (50\%) of individuals had both canal and otolith dysfunction (Group 3), four (29\%) of individuals had otolith only dysfunction (Group 2), and three (21\%) of individuals had canal only dysfunction (Group 1). These findings contribute to the growing body of knowledge that has found that involvement of different vestibular mechanisms occurs with $\mathrm{P}-\mathrm{VeD}[6,9,10,14]$. This information was paramount to the current investigation because it provided the foundation for the investigation's primary objective. Confirmation of the selective effect of pathology on canal and otolith mechanisms led to the expansion of the descriptors used by individuals with vestibular dysfunction and improved our understanding of how the unique functional contributions of canal and otolith mechanisms affects perceived orientation and balance ability after substrate damage.

In summary, when individuals report symptoms of dizziness due to suspected $\mathrm{P}-\mathrm{VeD}$, the qualitative nature can assist in deciding which vestibular mechanisms are involved. From the history, the focus should not only be on recognizing rotary vertigo as the descriptor related to $\mathrm{P}-\mathrm{VeD}$, but should also include words that reflect linear-type sensations. Expanding the descriptors to accept both rotary- and lineartype symptoms can facilitate identification of individuals that need vestibular function testing that includes measurement of both canal and otolith function. Furthermore, improved understanding of the involved vestibular mechanism may impact the implementation and success of rehabilitation. The distinct impairments found in individuals with canal versus otolith dysfunction also support further investigation to identify whether use of exercises strategies that optimally stimulate residual function specific to the involved vestibular mechanism would improve outcomes in VR.

There are several limitations and improvements that can be made with this preliminary report. Although there has been recent expansion of vestibular function testing, which includes testing of otoliths, there are limitations with all current available testing $[5,16]$. While caloric testing is a widely accepted as the "gold standard" for detecting unilateral vestibular loss and strongly recommended technique, it only tests a very low frequency range of vestibular function and there can also be a variability of response if there is inadequate irrigation [35]. The rotational chair uses head movement as the stimulus and provides stimulus frequencies that are higher as compared to caloric testing; however, the frequencies are on the lower end of normal head motion and the chair is less helpful in detecting unilateral lesions [35]. According to Schubert et al. [36], the horizontal head thrust (head impulse) 
test, which is used for identifying VOR dysfunction, has a sensitivity of $71 \%$ and specificity of $82 \%$. The authors questioned whether the variability of the sensitivity could be due to proficiency in performing the testing technique. With SVV testing there has been limited benefit from testing patients beyond the acute stage of $\mathrm{P}-\mathrm{VeD}$ because the results tend to normalize over time. However, when SVV is measured while applying vibration to the mastoid bone, as was used in this study, the sensitivity has been calculated as $91 \%$ and the specificity as 92\% [37]. With VEMP testing, reliability and validity of the responses is dependent on delivery of an adequate stimulus and with a consistent tonic contraction of the sternocleidomastoid muscle; however, the response can vary depending on the methodology used $[16,38]$. To address these potential problems, this study performed monitoring of electromyography (EMG) concurrently with the auditory stimulus and provided visual feedback to subjects to maintain uniform EMG during testing. Also, if an abnormal response to the auditory stimulus occurred, a bone stimulus to the mastoid process was performed to prevent a false positive due to possible stimulus conduction issues. The limitations with these vestibular function tests may have caused misclassification of vestibular dysfunction of some individuals. As the validity and reliability of testing improves, future studies can verify this study's findings. Also, to improve the DSI's usefulness in the clinic, future examination of the validity and reliability of this instrument should be performed on individuals with and without $\mathrm{P}-\mathrm{VeD}$ who report dizziness and imbalance.

The small sample size of this study limits the generalizability of conclusions drawn from these results. Additional investigations with larger sample sizes that include individuals with dysfunction of canal only, otolith only, and canal and otolith substrate are needed. Information from this type of study would support investigations that examine the use of exercise strategies that may optimally rehabilitate impairments of canal versus otolith dysfunction.

\section{Conflict of Interests}

The authors declare that there is no conflict of interests regarding the publication of this paper.

\section{Acknowledgments}

This work was supported by Florida Physical Therapy Association's Linda Crane Research Grant. The authors thank Dr. Simon Angeli, M.D., and Dr. Fred Telischi, M.D., from the Ear Institute of the University of Miami Miller School of Medicine, Miami, FL, for their referral of subjects.

\section{References}

[1] A. H. Clarke, "Laboratory testing of the vestibular system," Current Opinion in Otolaryngology and Head and Neck Surgery, vol. 18, no. 5, pp. 425-430, 2010.

[2] J. A. Goebel, "Practical anatomy and physiology," in Practical Management of the Dizzy Patient, pp. 3-16, Lippincott, Williams \& Wilkins, Philadelphia, Pa, USA, 2001.
[3] T. Tomanovic and J. Bergenius, "Different types of dizziness in patients with peripheral vestibular diseases their prevalence and relation to migraine," Acta Oto-Laryngologica, vol. 130, no. 9, pp. 1024-1030, 2010.

[4] T. Brandt and M. Strupp, "General vestibular testing," Clinical Neurophysiology, vol. 116, no. 2, pp. 406-426, 2005.

[5] G. M. Halmagyi, "Garnett Passe and Rodney Williams memorial lecture: new clinical tests of unilateral vestibular dysfunction," Journal of Laryngology and Otology, vol. 118, no. 8, pp. 589-600, 2004.

[6] U. SchÖnfeld, K. Helling, and A. H. Clarke, "Evidence of unilateral isolated utricular hypofunction," Acta Oto-Laryngologica, vol. 130, no. 6, pp. 702-707, 2010.

[7] T. Brandt, "Otolithic vertigo," Advances in Oto-RhinoLaryngology, vol. 58, pp. 34-47, 2001.

[8] J. G. Colebatch, G. M. Halmagyi, and N. F. Skuse, "Myogenic potentials generated by a click-evoked vestibulocollic reflex," Journal of Neurology Neurosurgery and Psychiatry, vol. 57, no. 2, pp. 190-197, 1994.

[9] S. Iwasaki, Y. Takai, K. Ito, and T. Murofushi, "Abnormal vestibular evoked myogenic potentials in the presence of normal caloric responses," Otology and Neurotology, vol. 26, no. 6, pp. 1196-1199, 2005.

[10] P. Monstad, S. Økstad, and A. Mygland, "Inferior vestibular neuritis: 3 cases with clinical features of acute vestibular neuritis, normal calorics but indications of saccular failure," BMC Neurology, vol. 6, article 45, 2006.

[11] B. S. Shin, S. Y. Oh, J. S. Kim et al., "Cervical and ocular vestibular-evoked myogenic potentials in acute vestibular neuritis," Clinical Neurophysiology, vol. 123, no. 2, pp. 369-375, 2012.

[12] D. Basta, I. Todt, H. Scherer, A. Clarke, and A. Ernst, "Postural control in otolith disorders," Human Movement Science, vol. 24, no. 2, pp. 268-279, 2005.

[13] S. C. A. Hegemann and A. Palla, "New methods for diagnosis and treatment of vestibular diseases," F1000 Medicine Reports, vol. 2, no. 1, article 60, 2010.

[14] M. Karlberg, M. Annertz, and M. Magnusson, "Acute vestibular neuritis visualized by $3-\mathrm{T}$ magnetic resonance imaging with high-dose gadolinium," Archives of Otolaryngology_Head and Neck Surgery, vol. 130, no. 2, pp. 229-232, 2004.

[15] Y. Valko, S. C. A. Hegemann, K. P. Weber, D. Straumann, and C. J. Bockisch, "Relative diagnostic value of ocular vestibular evoked potentials and the subjective visual vertical during tilt and eccentric rotation," Clinical Neurophysiology, vol. 122, no. 2, pp. 398-404, 2011.

[16] F. L. Wuyts, J. Furman, R. Vanspauwen, and P. van de Heyning, "Vestibular function testing," Current Opinion in Neurology, vol. 20, no. 1, pp. 19-24, 2007.

[17] G. M. Halmagyi, S. T. Aw, M. Karlberg, I. S. Curthoys, and M. J. Todd, "Inferior vestibular neuritis," Annals of the New York Academy of Sciences, vol. 956, pp. 306-313, 2002.

[18] C. M. Kingma and H. P. Wit, "Asymmetric vestibular evoked myogenic potentials in unilateral Menière patients," European Archives of Oto-Rhino-Laryngology, vol. 268, no. 1, pp. 57-61, 2011.

[19] C. de Waele, P. T. B. Huy, J. Diard, G. Freyss, and P. P. Vidal, "Saccular dysfunction in Meniere's disease," The American Journal of Otology, vol. 20, no. 2, pp. 223-232, 1999.

[20] L. Manzari, A. R. Tedesco, A. M. Burgess, and I. S. Curthoys, "Ocular and cervical vestibular-evoked myogenic potentials to 
bone conducted vibration in Ménière's disease during quiescence versus during acute attacks," Clinical Neurophysiology, vol. 121, no. 7, pp. 1092-1101, 2010.

[21] Y. H. Young, T. W. Huang, and P. W. Cheng, "Assessing the stage of Ménière's disease using vestibular evoked myogenic potentials," Archives of Otolaryngology-Head and Neck Surgery, vol. 129, no. 8, pp. 815-818, 2003.

[22] S. Tabak, H. Collewijn, and L. J. J. M. Boumans, "Deviation of the subjective vertical in long-standing unilateral vestibular loss," Acta Oto-Laryngologica, vol. 117, no. 1, pp. 1-6, 1997.

[23] J. H. J. Allum and N. T. Shepard, "An overview of the clinical use of dynamic posturography in the differential diagnosis of balance disorders," Journal of Vestibular Research: Equilibrium and Orientation, vol. 9, no. 4, pp. 223-252, 1999.

[24] R. W. Baloh and V. Honrubia, "The peripheral vestibular system," in Clinical Neurophysiology of the Vestibular System, pp. 20-43, F.A. Davis, Philadelphia, Pa, USA, 1990.

[25] C. H. Markham, "Vestibular control of muscular tone and posture," Canadian Journal of Neurological Sciences, vol. 14, no. 3, pp. 493-496, 1987.

[26] V. J. Wilson and B. W. Peterson, "Peripheral and central substrates of vestibulospinal reflexes," Physiological Reviews, vol. 58, no. 1, pp. 80-105, 1978.

[27] S. J. Herdman, "Vestibular rehabilitation," CONTINUUM Lifelong Learning in Neurology, vol. 12, no. 4, pp. 151-167, 2006.

[28] A. Ernst, D. Basta, R. O. Seidl, I. Todt, H. Scherer, and A. Clarke, "Management of posttraumatic vertigo," Otolaryngology-Head and Neck Surgery, vol. 132, no. 4, pp. 554-558, 2005.

[29] D. E. Krebs, K. M. Gill-Body, S. W. Parker, J. V. Ramirez, and M. Wernick-Robinson, "Vestibular rehabilitation: useful but not universally so," Otolaryngology-Head and Neck Surgery, vol. 128, no. 2, pp. 240-250, 2003.

[30] J. C. Enticott, J. J. Vitkovic, B. Reid, P. O’Neill, and M. Paine, "Vestibular rehabilitation in individuals with inner-ear dysfunction: a pilot study," Audiology and Neurotology, vol. 13, no. 1, pp. 19-28, 2007.

[31] G. P. Jacobson and C. W. Newman, "The development of the dizziness handicap inventory," Archives of OtolaryngologyHead and Neck Surgery, vol. 116, no. 4, pp. 424-427, 1990.

[32] S. L. Whitney, D. M. Wrisley, K. E. Brown, and J. M. Furman, "Is perception of handicap related to functional performance in persons with vestibular dysfunction?" Otology and Neurotology, vol. 25, no. 2, pp. 139-143, 2004.

[33] J. M. Serrador, L. A. Lipsitz, G. S. Gopalakrishnan, F. O. Black, and S. J. Wood, "Loss of otolith function with age is associated with increased postural sway measures," Neuroscience Letters, vol. 465, no. 1, pp. 10-15, 2009.

[34] K. J. Murray, K. D. Hill, B. Phillips, and J. Waterston, "The influence of otolith dysfunction on the clinical presentation of people with a peripheral vestibular disorder," Physical Therapy, vol. 87, no. 2, pp. 143-152, 2007.

[35] T. D. Fife, R. J. Tusa, J. M. Furman et al., "Assessment: vestibular testing techniques in adults and children-report of the therapeutics and technology assessment subcommitte of the American academy of neurology," Neurology, vol. 55, no. 10, pp. 1431-1441, 2000.

[36] M. C. Schubert, R. J. Tusa, L. E. Grine, and S. J. Herdman, "Optimizing the sensitivity of the head thrust test for identifying vestibular hypofunction," Physical Therapy, vol. 84, no. 2, pp. 151-158, 2004.
[37] M. Karlberg, S. T. Aw, G. M. Halmagyi, and R. A. Black, "Vibration-induced shift of the subjective visual horizontal: a sign of unilateral vestibular deficit," Archives of Otolaryngology-Head and Neck Surgery, vol. 128, no. 1, pp. 21-27, 2002.

[38] F. W. Akin, O. D. Murnane, P. C. Panus, S. K. Caruthers, A. E. Wilkinson, and T. M. Proffitt, "The influence of voluntary tonic EMG level on the vestibular-evoked myogenic potential," Journal of Rehabilitation Research and Development, vol. 41, no. 3, pp. 473-480, 2004. 


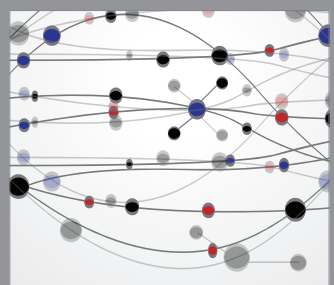

The Scientific World Journal
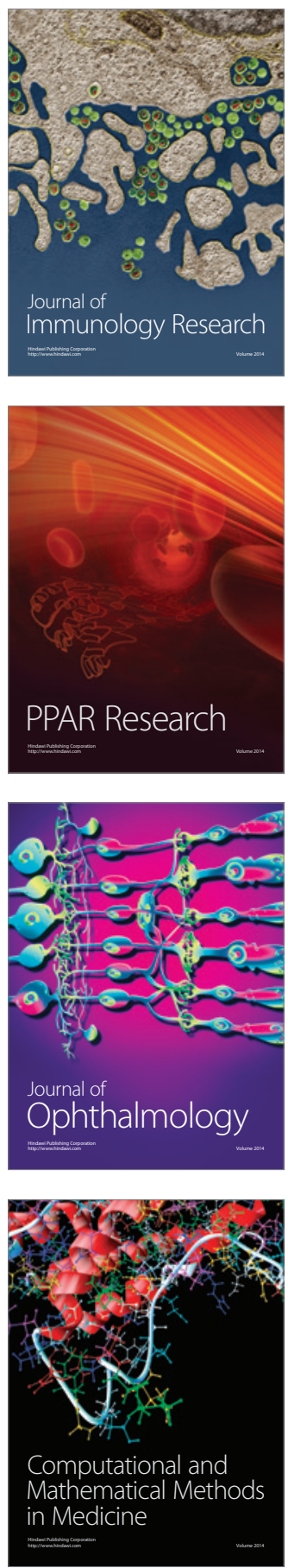

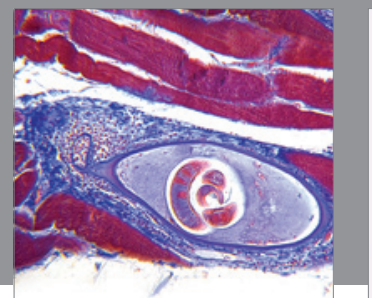

Gastroenterology

Research and Practice
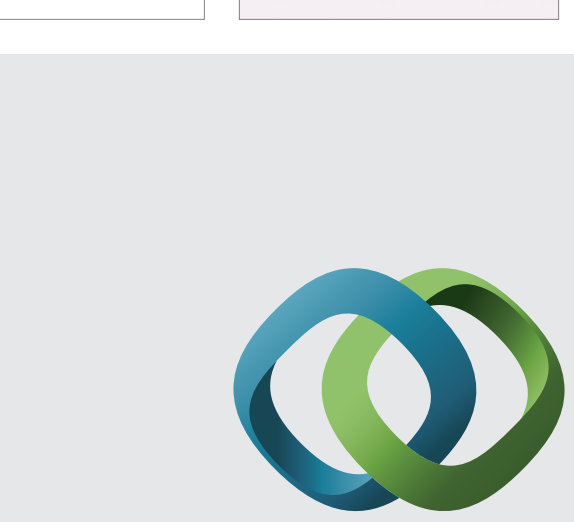

\section{Hindawi}

Submit your manuscripts at

http://www.hindawi.com
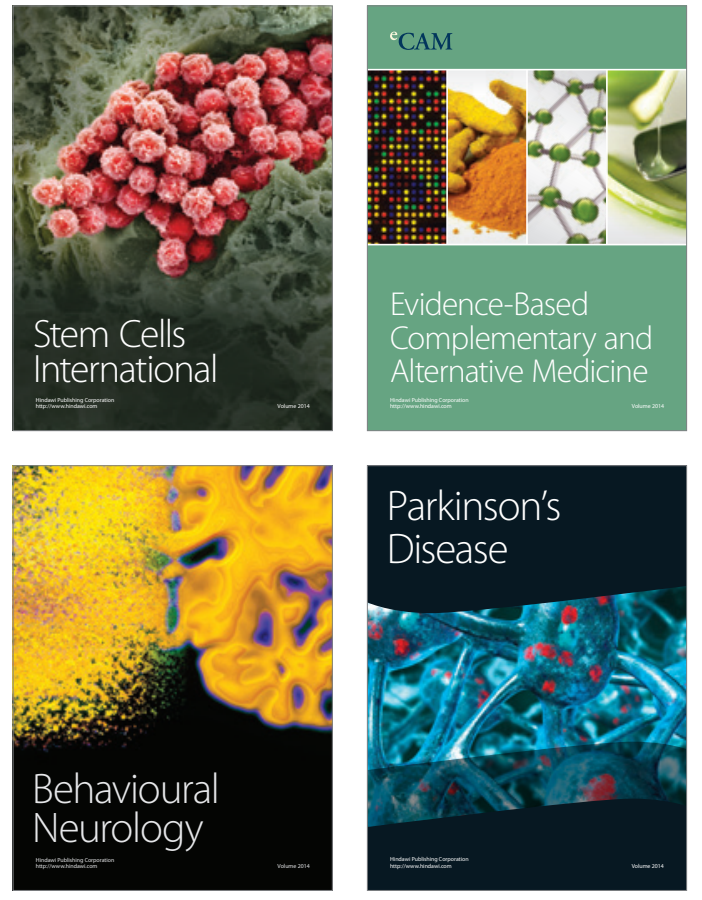
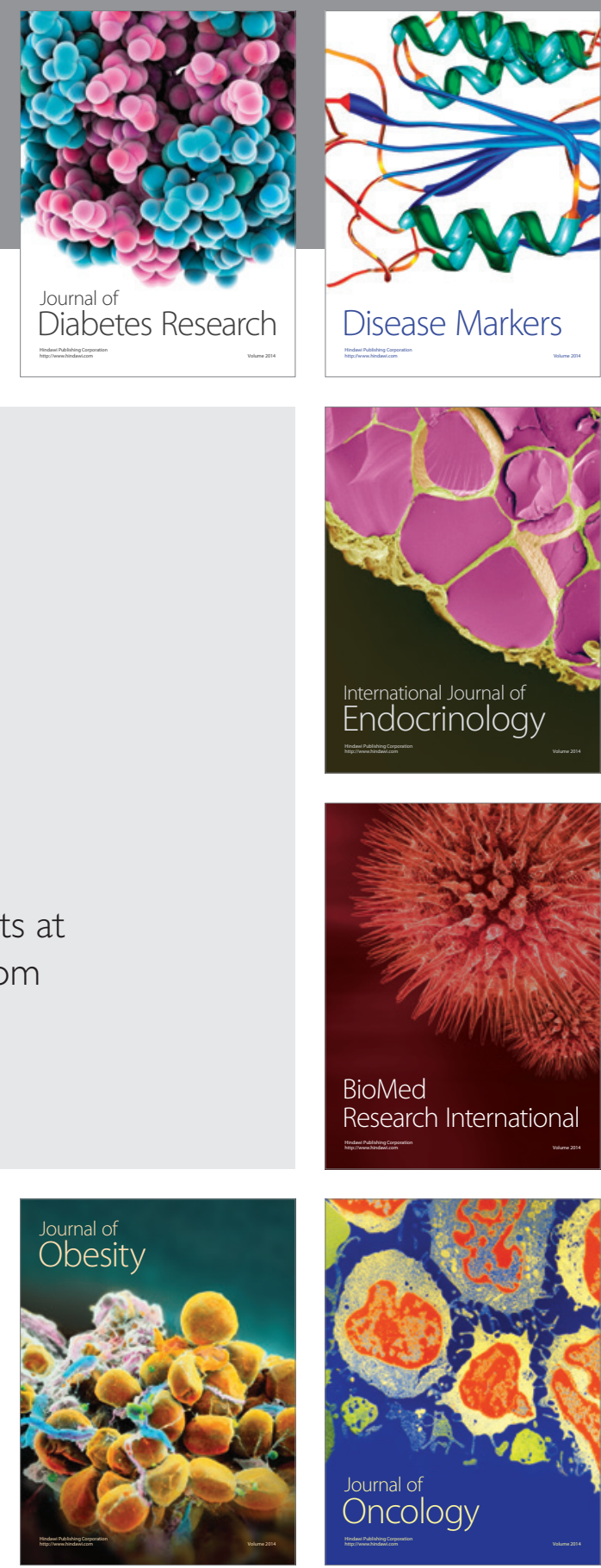

Disease Markers
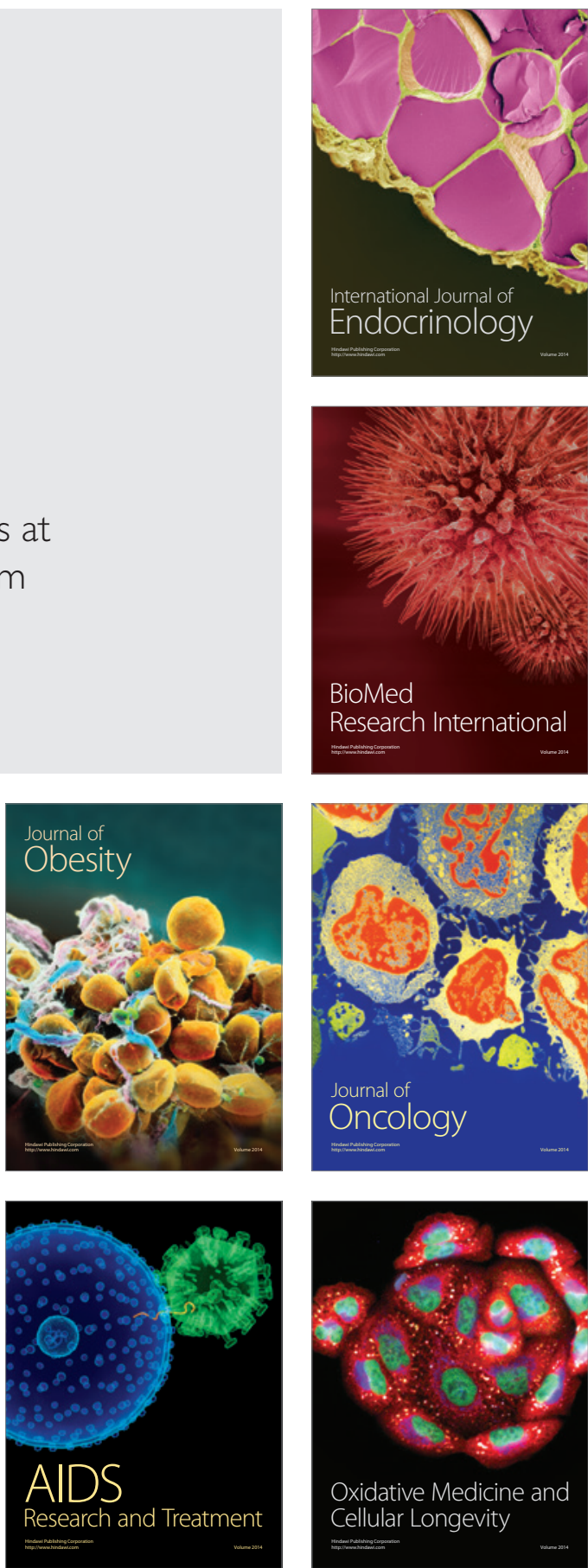